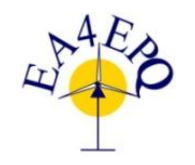

International Conference on Renewable Energies and Power Quality

(ICREPQ' 13)

Bilbao (Spain), $20^{\text {th }}$ to $22^{\text {th }}$ March, 2013

$R$ enewableE nergy and P ower $Q$ uality J ournal (RE\&PQJ)

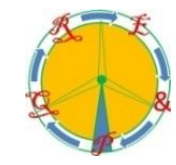

ISSN 2172-038 X, No.11, March 2013

\title{
Distributed Generation Penetration Impact on Distribution Networks Loss
}

\author{
A. Sheikhi ${ }^{1}$, A. Maani ${ }^{2}$, F.Safe ${ }^{3}$, A.M. Ranjbar ${ }^{4}$ \\ 1,2,3,4 Department of Electrical Engineering \\ SharifUniversity of Technology \\ Tehran, Iran \\ ${ }^{1}$ Phone/Fax number:+989126613840, e-mail: ${ }^{1}$ sheikhi@ee.sharif.edu,${ }^{2}$ maani@ee.sharif.edu, ${ }^{3}$ Kharati@ee.sharif.edu, \\ amranjbar@sherif.edu
}

\begin{abstract}
Cost of annual energy loss occurs in distribution networks is an important issue in distribution systems planning, design and operation $\&$ discos are pleased to be informed about financial worth of loss extract from their profit to evaluate economic feasibility of improvement projects. there are several dominating factors involved in distribution loss cost evaluation, but most of the times price of loss occurs in distribution systems is calculated regardless of location, consumption pattern and etc, but recent changes in Iran distribution power system including removing energy subsidies, deregulation, increase in distributed generation units and trend toward loss reduction had been resulted in much more importance to have actual price of distribution network losses.

In this paper, Distributed generation units penetration level influence on distribution networks worth of loss is investigated, power delivered to distribution systems from these resources is directly connected to loads and no transmission cost is involved, in the proposed formulation this difference between centralized and decentralized generation is covered. It is shown that distribution worth of loss follow a U-shape trajectory by increase in distributed generation units' penetration level.
\end{abstract}

\section{Key words}

Distribution network, loss cost, distributed generation (DG), penetration level.

\section{Introduction}

Distribution systems although are the most visible part of power system and largest investment, maintenance and operation expense are pointed to these section, but they had not received the technological impact in same manner as the generation \& transmission systems. Most of them are operated with least monitoring \& automation systems [1]. In Iran distribution networks, $17.1 \%$ of total system energy generation is estimated to be lost in these networks; the amount of power loss at peak hours is greater than this percent. In recent years there is a growing trend in distribution utilities to reduce system losses and improve total system efficiency [2]. Installation and operation of DG units is one of the regulators' politics in recent years to reduce system losses and improve system efficiency. Government had developed some facilities to support investors to establish DG units as one of the most important loss reduction and peak shaving programs to improve system efficiency and reduce peak load. So, there is a growing trend in Iran among buildings owners to install and operate distributed generation stations especially Combined Heat \& Power (CHP) units due to facilities government had been assigned to commercial buildings. By considering this point which Iran is a developing country and need more generation power, Iran government decided to install about 6300 MW Combine Heat and Power (CHP) units until 2016 [3]. To implement this plan, some politics have been implemented in Iran like assigning subsided for CHP building and its required fuels. By this tension to CHP it will be important to consider its effect in loss and also worth of loss in the customer and generation companies view.

High levels of penetration of DG are new challenge for Iran distribution utilities [4]. Power injected from these units to distribution feeders change network power flow modifying annual energy loss and cost of providing this loss. Although it is considered that DG reduce system energy losses, it is shown that annual energy loss in distribution networks varies as a U-shape trajectory by increase in DG penetration level [4], regulators attempt is to set facilities in way to correspond DG installed capacity in curve minimum point. 
In this paper instead of annual energy loss, cost of supplying annual energy loss is investigated which has much more importance in comparison to annual energy loss for utilities and consumers. In annual energy loss evaluation, energy loss is computed regardless of cost of providing these losses, but power delivered to distribution feeders is supplied by transmission grid and DG units installed in feeder length, cost of providing first is generation cost plus transmission cost while at second, since DG units are directly connected to loads, there is no transmission cost associated with them. This difference is covered in present paper proposed formulation.

Grid dispatch which is determined by optimal power flow in vertically integrated utilities and by power markets in restructured systems is a dominating factor which determines the flow of electricity in a transmission network. Utilizing power trace methods, the contribution of each generating station in supplying system loads and contribution of each load at transmission lines cost can be estimated. Since generating stations and transmission costs differ from station to station and line to line according to system technical characteristics, many factors are involved in distribution networks worth of loss evaluation. Network dispatch, location, consumption pattern, system investment costs are the most important parameters in distribution network loss cost which are presented in section III.

Distribution system operators are pleased to know the financial worth of loss occurs in their networks, they need to know the distribution network loss cost to evaluate improvement projects economic feasibility, setting priority of networks in implementing improvement projects and to know the financial flow of energy in their network. So annual energy loss and annual energy loss cost are the most important technical and economical issues in distribution networks operation respectively.

Several studies have been conducted to assess losses variations and/or to allocate such variations between DG and consumers. Reference [5] did pinpoint the importance of losses variations for DG connection costs and proposed a methodology, based on second-order sensitivities, to compute such losses variations. However, this method is only valid for small DG penetration variations, as it will be proved later in this paper. References [6] and [7] proposed different methods to compute marginal nodal losses in order to provide economic signals to both consumers and DG. Reference [8] made the analysis of predicting how losses will vary when connecting a specific photovoltaic plant to a specific feeder, based on a feeder section-bysection analysis. Other authors analyzed some other aspects, as the importance of the location of DG for losses reduction [9]-[11], even proposing in some cases some optimization algorithm to find the better DG location. How the control of the reactive power injected by DG plants impact losses levels was studied in [12] and [13]. A more complete study was carried out in [14], where impact of operational aspects on losses variations and acceptable DG penetration levels are analyzed, based on a specific case study. Reference [15] investigates the impact of distributed generation on distribution losses.

Such valuable studies can help distribution utilities to set laws which result in desired point of DG penetration level.

The paper is organized as follow, in section II among all DG impacts on distribution systems, impacts on distribution network loss is highlighted, distribution networks worth of evaluation process loss is discussed in section III, in section IV loss cost trajectory with increase in DG penetration level is formulated and in section $\mathrm{V}$ proposed formulation is applied to test network. Paper concludes in section VI by summary and conclusion.

\section{DG Impacts on Distribution Systems}

At first some useful definition which has been applied to this paper is mentioned as follow:

1) DG installed capacity: The total maximum output of each DG unit. The DG installed capacity in the network is the sum of the individual DG installed capacities.

2) Capacity factor: The ratio of the energy produced, during the period of time considered, to the energy that could have been produced if DG would have operated at continuous full power during the same time period.

3) DG penetration level: The ratio of the amount of DG energy injected into the network to the feeder capacity [5].

DGPenetration level $=\frac{\text { Capacity Factor } \text {. Installed Capacity }}{\text { Feeder Capacity }}(1)$

Distributed Energy Resources (DER) had changed many aspects of distribution system operation, design and implementation. By increasing more decentralized systems with smaller generating units connecting directly to the distribution networks near demand consumption and consider distribution companies to reduce loss in their networks and benefit its results finding optimum point of cost of supplying loss in power distribution networks.

To analyze the impact of DG on losses one single DG plant (a CHP unit) was located in several nodes along the feeder.

Note that a CHP unit was simulated by an hourly generation profile obtained from recorded real annual hourly data from a set of CHP plants in Iran.

To model the issue, the radial IEEE 34-node test feeder is presented in this paper [4]. (see figure 1)

In order to compute energy losses on an annual basis, a load flow algorithm (in this paper the N-R load flow has been applied) should be run each hour of the year. Annual losses are calculated as the sum of hourly energy losses and to analyze the impact of DG energy 
production on energy losses, DG was modeled as a negative PQ load with unit power factor.

The single DG's result (DG connected to the node number 848) annual losses variation as a function the penetration level of it like U-shape trajectory. Losses after a minimum value start to increase with higher DG penetration levels. Results of simulations and evaluation of cost of providing losses when feeder is fed by node 15 of IEEE 30-bus test network at different DG penetration levels are provided in sectionV.

\section{Distribution Networks Loss-Cost Evaluation}

Financial worth of $1 \mathrm{kWh}$ energy loss occurs in distribution network is equal to increment in distribution utility profit when annual energy loss in decreased by 1 kwh. So to determine the worth of loss, recent $\mathrm{kWh}$ should be traced and contribution of each generating station and transmission lines on supplying this energy loss should be evaluated.

There are many significant factors influencing the actual distribution networks worth of loss, the complexity of these factors often prevent them to be involved in practical applications, such as billing strategies, loss reduction projects economic evaluation and etc.

In this section most important parameters affecting distribution networks loss are listed,

A. Location

Location of distribution network plays a key role in worth of loss occurs; there is a considerable difference between two networks, one in vicinity of a generating station, other in thousands of kilometers far away. For later, the power delivered to distribution network had been passed through long transmission lines and thousands of dollars associated with it. Transmission cost is calculated with MW-mile method which covers both power and location of distribution network.

\section{B. Consumption time pattern}

The way electric loads demand power from the distribution network affect the time pattern of demanded power from centralized generating stations, transmission grid and distributed generation units, generation technology and transmission costs vary with change in time pattern.

\section{Network Power Dispatch}

Dispatch of power shows that power demanded by each load is supplied by generating stations which different generation costs can influence the distribution networks worth of loss.

D. Supplying generation units

Cost of producing power generated in centralized generating stations varies strongly with generation technology, both for capital and operating costs,

\section{E. Linking Transmission lines costs}

Transmission lines of length of kilometers long had been implemented by huge amount of capital costs; also they have different efficiencies, capacity ...

They can influence the price of power delivered to distribution substation.
F. Supplying distributed generation units costs Small distributed energy supply station had been gathered a large amount of attention during recent decade, they are going to play a key role in future energy resources, they operate usually in vicinity of electric loads, so power generated by them is more likely to be cheap in comparison of centralized generating stations, since power flown in distribution networks is impressed by DGs, in proposed formulation the distributed generation units costs, both capital and operating ones are modeled. This factor is covered in proposed formulation presented in section II, percent of losses provided by grid costs for generation and transmission of power, but percent supplied by DG unit's costs only for generation costs.

\section{G. Reactive power sources}

Some portion of reactive power demanded by electric loads is supplied by reactors and capacitor banks installed in substation units, top of distribution poles, Bialek at [16], had developed a simple methodology based on proportional sharing to trace the flow of electricity in distribution and transmission network and contribution of each source in supplying each load and contribution of each load at transmission line losses can be determined utilizing this method. In this section this method is used to evaluate distribution system worth of loss and to analyze how it changes with increase in distributed generation penetration level.

Units' generation costs can be obtained by averaging generating stations operating expenses. About transmission costs, a simple method, MW-Mile is used to evaluate cost of transmitting power from centralized generation units to load points, for decentralized units, there is no transmission cost associated with them [17].

\section{Loss-Cost trajectory By Changeing DG Penetration Level}

It is shown at [4] that distribution networks annual energy loss change with increase in various distributed generation technologies like a U- shape trajectory. In this paper instead of annual energy loss, total distribution networks worth of loss is investigated which has much more importance than annual energy loss for distribution utilities regulators and operators.

It is necessary to consider the source of power dissipated in distribution networks generation costs, power delivered to feeder by transmission grid had been passed through long distance transmission lines, but about distributed generating stations, the power produced by these units is consumed in the vicinity of generators, so the transmission line losses and transit right should be included in the loss cost computations to cover the difference between these two types of generation. In this section, influence of increase in DG penetration level on distribution networks worth of loss is evaluated.

Distribution network worth of loss is formulated as summation of generation and transmission costs as equation 2 ,

Loss cost $=\left(\operatorname{cost}_{\mathrm{Gen}}+\cos _{\mathrm{trans}}\right)_{\mathrm{Grid}}+\left(\operatorname{cost}_{\mathrm{Gen}}\right)_{\mathrm{DGs}}(2)$ 
Distribution network loss was traced and contribution of grid and each DG unit in supplying this amount of annual energy losses was evaluated, by summation of distributed generation units production cost the second term in equation 2 can be computed, for first term it is necessary to trace power delivered to distribution network and determine the contribution of each centralized station in supplying the feeder demand. Centralized units generation cost then is computed, about transmission costs, several transmission cost allocation had been developed to date, in this paper MW-Mile method is used which has simple implementation for large scale grids [17].

Centralized units generation cost can be organized by equation 3.

$\operatorname{cost}_{\mathrm{Gen}}=\sum_{\mathrm{i}=1}^{\mathrm{n}} \mathrm{E}_{\mathrm{i}} \cdot \mathrm{C}_{\mathrm{i}}$

In this equation,

$E_{i}$, Denotes for energy loss allocated to generator i,

$C_{i}$, Is average generation cost of $\mathrm{i}^{\text {th }}$ generation unit.

$\mathrm{n}$, centralized generation stations number.

Transmission cost can be computed by MW-mile method [17] as presented in equation 4 ,

$\operatorname{cost}_{\text {trans }}=A_{i} \cdot \sum_{i} C_{i} \cdot \frac{\sum_{\boldsymbol{i}} \boldsymbol{l}_{\boldsymbol{i}} \cdot \Delta \boldsymbol{P}_{\boldsymbol{i}, t}}{\sum_{\boldsymbol{i}} \boldsymbol{l}_{\boldsymbol{i}}\left|\boldsymbol{P}_{\boldsymbol{i}, t}\right|}$

In this equation,

$\mathrm{t}$, denotes time,

$l_{i}$, denotes for transmission line length,

$P_{i}$, is transmission line power carried,

$C_{i}$, is transmission cost per unit energy,

$A_{i}$, is a constant.

Distributed energy resources production cost can be computed as equation 5 ,

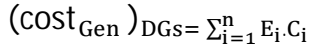

As Distributed Generation penetration level increase, distributed generation resources contribution in supplying system losses increase and consequently transmission cost decrease, centralized generating cost decrease and cost due to generation at distributed resources grows up. Distribution network loss cost which is summation of these parameters should be minimized by change in DG penetration level.

Dependent of system technical characteristics such as generating stations technology and operating costs, transmission network and other factors, total system loss cost vary with change in Distributed Generation penetration level.

\section{Case Study}

The proposed formulation about distributed generation penetration level influence on loss cost was applied to several medium voltage distribution systems with different conditions, all results were qualitatively similar. In this section simulation results of applying proposed formulation to IEEE 34-bus test feeder are illustrated.
Feeder single line diagram and its basic technical information's are provided in the figure 1and table (I) respectively [18].

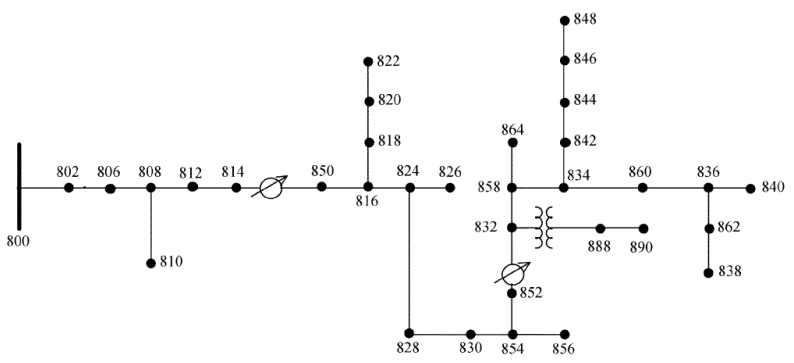

Fig 1. Single-line diagram of the IEEE 34-node test feeder.

Table I. IEEE 34-Node test feeder basic data

\begin{tabular}{cc}
\hline Voltage & $24.9 \mathrm{KV}$ \\
Feeder Capacity & $713.1 \mathrm{KVA}$ \\
Length & $93.9 \mathrm{Km}$ \\
\hline
\end{tabular}

To evaluate grid production cost, feeder's node 800 is assumed to be connected to node 15 of IEEE 30-bus test transmission network (see figure 2).

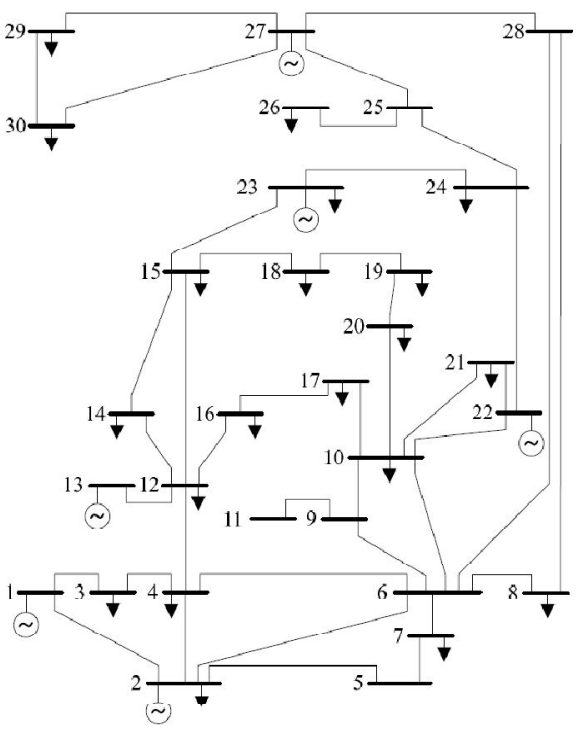

Fig. 2. The 30- bus IEEE test system single line diagram

As mentioned before, for DG unit, a CHP system was placed in different nodes and similar results obtained, in this section node 848 of distribution network is selected to place DG unit. DG unit was modeled as a negative PQ load with a unity power factor. Capacity factor of CHP systems is 0.5 [4], DG installed capacity is increased from 0 to $114.1 \mathrm{KVA}$ which is equivalent to penetration level increase from 0 to $8 \%$.

Iran distribution network typical loads were divided into three levels, light, medium and heavy whose duration is 
provided in the table (II) to compute annual energy loss cost [19].

Table II. Test feeder load duration table

\begin{tabular}{c|cc}
\hline Load level & $\begin{array}{c}\text { Percent of } \\
\text { peak load }\end{array}$ & Annual duration \\
\hline Light & 35 & 35064 \\
Medium & 65 & 24545 \\
Heavy & 95 & 105193 \\
\hline
\end{tabular}

Generators production cost is summarized in tables (III) and (IV) respectively for centralized and decentralized units.

Table III. Centralized Generating Units Production Cost

\begin{tabular}{||c|c|c|c|c||}
\hline & BUS & \multicolumn{3}{|c|}{ LOAD LEVEL } \\
\hline Cost of & Number & Light & Medium & Heavy \\
\cline { 2 - 5 } generation & 1 & 0.025 & 0.030 & 0.04 \\
\cline { 2 - 5 }$(\$ /$ kwh $)$ & 2 & 0.025 & 0.030 & 0.042 \\
\cline { 2 - 5 } & 13 & 0.020 & 0.035 & 0.04 \\
\hline
\end{tabular}

Table IV. Decentralized Generating Units Production cost

\begin{tabular}{||c|c|c|c||}
\hline \multirow{2}{*}{} & \multicolumn{3}{|c||}{ LOAD LEVEL } \\
\cline { 2 - 4 } & Light & Medium & Heavy \\
\hline $\begin{array}{c}\text { Cost of } \\
\text { generation } \\
(\$ / \mathrm{kwh})\end{array}$ & 0.040 & 0.045 & 0.06 \\
\hline
\end{tabular}

Transmission cost is assumed to be constant for all lines per Mw-mile and according to table V.

Table V. transmission cost

\begin{tabular}{||c|c|c|c||}
\hline \multirow{2}{*}{} & \multicolumn{3}{|c||}{ LOAD LEVEL } \\
\cline { 2 - 4 } & Light & Medium & Heavy \\
\hline $\begin{array}{c}\text { Cost of } \\
\text { transmission } \\
(\$ / \mathrm{kw} . \mathrm{km})\end{array}$ & 0.00015 & 0.00015 & 0.00020 \\
\hline
\end{tabular}

Flow of electricity in transmission and distribution network was traced and Simulation programs with different penetration levels run, for each simulation, the contribution of each generating station and transmission lines in providing annual energy losses for test feeder loads was evaluated, then transmission cost, centralized generation cost and DG units production cost trajectory with increase in DG penetration level was evaluated, total test distribution network loss cost which is summation of all above parameters was also evaluated for each step. Results for centralized generation costs, transmission cost and DG production cost and total distribution network loss cost are provided in figures 3,4,5,6 respectively.

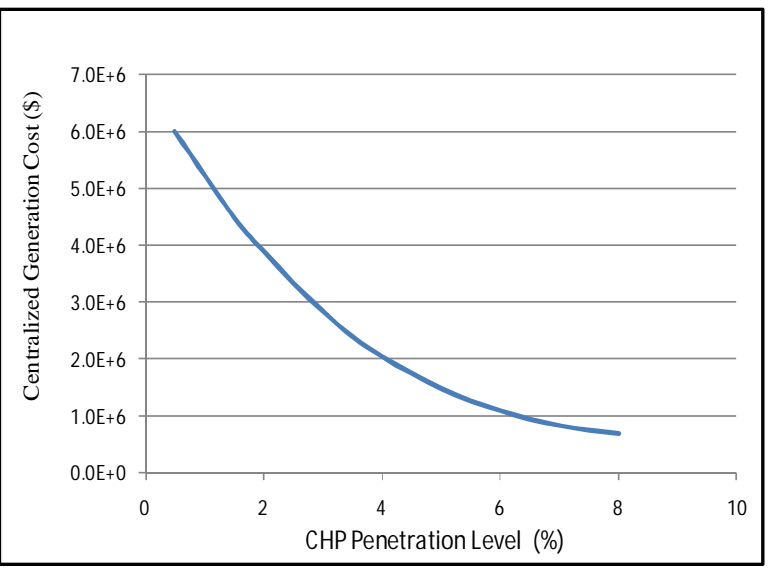

Fig 3.centralized generation cost trajectory

As DG penetration level increases, contribution of centralized generating stations in supplying system losses decrease.

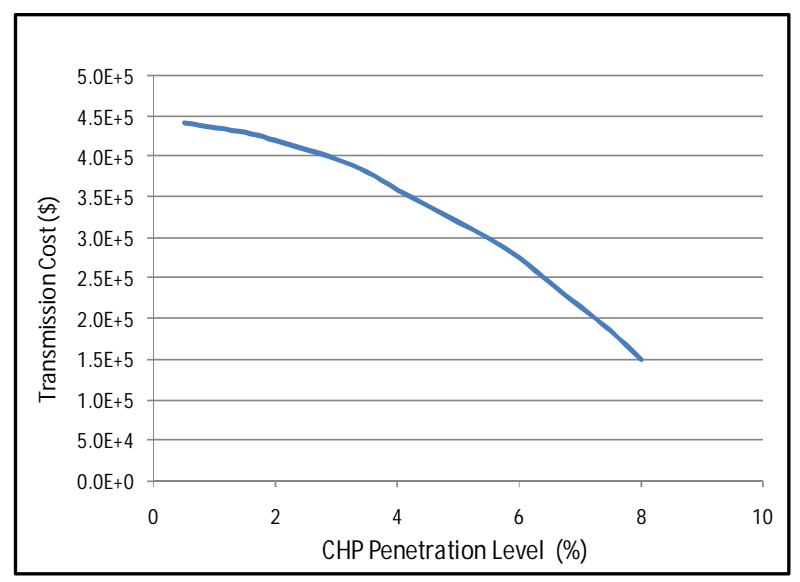

Fig 4.transmission cost trajectory

Increase in DG penetration level, reduce transmission lines loading and decrease transmission cost in MW-Mile method directly.

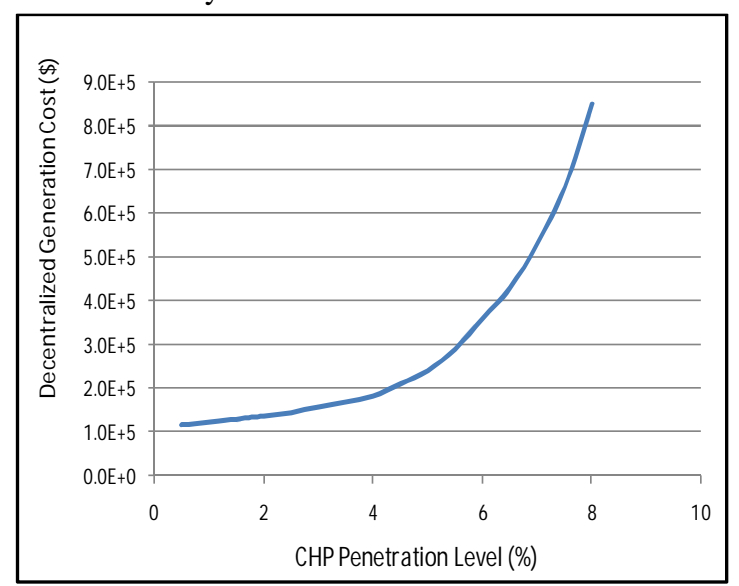

Fig 5.decentralized generation cost trajectory 


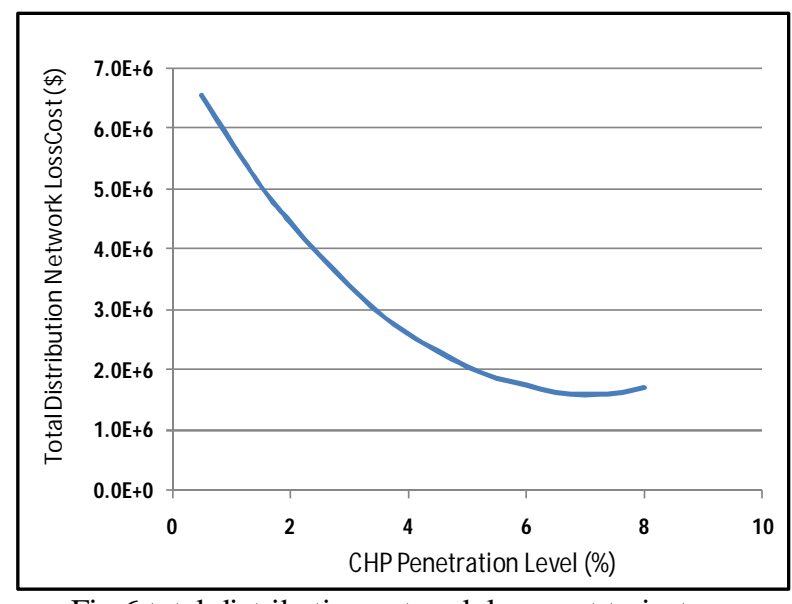

Fig 6.total distribution network loss cost trajectory

\section{Conclusion}

As DG penetration level increase, DG contribution in supplying feeder loads increase and consequently DG production cost increase, on the other hand increase in DG penetration level reduce centralized generation costs and transmission costs. Total distribution network loss cost which is summation of these factors has a U- shape trajectory whose minimum occurs in penetration level of $6.8 \%$.

\section{Acknowledgement}

Authors strongly appreciate from financial support of Elite National Foundation, Tehran, Iran for its financial support during the research.

\section{References}

[1] Aras Sheikhi, Mehdi S. Naderi, Ali Mohammad Ranjbar, G.B. Gharehpetian, Farahbakhsh Safe, "CHP Optimized Selection Methodology for a Multi-Carrier Energy System" International Review of Electrical Engineering (IREE), Vol.6, No. 4, Part B, June 2011

[2] Iran Energy annual Report 2009(page 14)

[3] CHP development plan in Iran(pages 2, 35)

[4] A. Sheikhi, A.M. Ranjbar, H. Oraee, "Financial analysis and optimal size and operation for a multicarrier energy system" Energy and Buildings, Elsevier, 2012.

[5] J. Mutale, G. Strbac, S. Curcic, and N. Jenkins, "Allocation of losses in distribution systems with embedded generation," Proc. Inst. Elect. Eng.,

Gener.,Transm., Distrib., vol. 147, no. 1, pp. 7-14, Jan. 2000.

[6] P. M. Costa and M. A. Matos, "Loss allocation in distribution networkswith embedded generation," IEEE Trans. Power Syst., vol. 19, no. 1, pp.384-389, Feb. 2004.

[7] T. Hoff and D. S. Shugar, "The value of grid-support photovoltaics inreducing distribution system losses," IEEE Trans. Energy Convers., vol.10, no. 3, pp. 569576, Sep. 1995.

[8] P. P. Barker and R.W. de Mello, "Determining the impact of distributedgeneration on power systems:
Part 1-radial distribution systems," inProc. IEEE Power Eng, Soc. Summer Meeting, Seattle, WA, 2000.

[9] W. El-Khattam and M. M. A. Salama, "Impact of distributed generationon voltage profile in deregulated distribution system," in Proc. PowerSystem Conf., Clemson, SC, 2002.

[10] T. Griffin, K. Tomsovic, D. Secrest, and A. Law, "Placement of dispersed generations systems for reduced losses," in Proc. 33rd Hawaii Int. Conf.System Sciences, 2000.

[11] P. Chiradeja and R. Ramakumar, "Benefits of distributed generation. Asimple case study," in Proc. 32nd Annu. Frontiers Power Conf., 1999.

[12] S. Persaud, B. Fox, and D. Flynn, "Impact of remotely connected windturbines on steady state operation of radial distribution networks," Proc.Inst. Elect. Eng., Gener.,Transm., Distrib., vol. 147, no. 3, pp. 157-163,May 2000.

[13] S. N. Liew and G. Strbac, "Maximizing penetration of wind generationin existing distribution networks," Proc. Inst. Elect. Eng., Gen., Transm.,

[14] J. Bialek, "Tracing the flow of electricity," Proc. Inst. Elect. Eng. Gen., Transm.,Distrib., vol. 143, pp. 313-320, July 1996.

[15] K. L. Lo, M. Y. Hassan, S. Jovanonic, "Assessment of MW-mile method for Pricing Transmission Services:A Negative Flo-Sharing Approach", ,IET Gen. Transm. Distrib. 2007, 1, (6), pp 904-911.

[16] IEEE test feeders, Washington University, available at, http://ee.washington.edu/testfeeders.

[17] R.Hooshmand and M.Ataei, "Optimal Capacitor Placement In Radial Actual Configuration And Operational Conditions of Distribution Systems Using RCGA", Journal of ELECTRICAL ENGINEERING, vol 58, no.4,189-199, 2007. 